\title{
La acción reflexiva en educación: buscando lo sustancial
}

\author{
Miguel A. SANTOS Rego \\ Universidad de Santiago de Compostela
}

\begin{abstract}
SUMMARY.-The first purpose of this study is to explore the main questions and topics connected with the recuperation of the «reflectivity» in education. Also, we pretend to inquiry into the most substantial aspects that accompany such recuperation in moments as live today, characterized by change and educational reform.

Nowadays, the great emphasis in the notion of reflection has relation with the rise of qualitative research about the study of educational processes; without to forget the sharp load of situational and / or cultural factors in its treatment. Of course, this involve theoretical and methodological changes.

On the other hand, we pay special attention to the process of reflection itself and some approaches post- Dewey. In the same way, we relate the topic with the professional education of teachers.

Finally, the cognitive, critical, and narrative elements included in the process of reflection are traced and developed; considering a comprehensive framework of educational improvement for our time.
\end{abstract}

\section{INTRODUCCIÓN}

Hoy asistimos en el mundo de la educación a una inusitada llamada a la reflexión, reclamo que llega a tener tintes sorprendentes cuando se hace transmitiendo una idea de radical novedad para la escuela, y no tanto como renovado y firme alegato en favor de uno de los más preclaros fines y logros a procurar en la formación humana. De todos modos, es bueno saludar con entusiasmo controlado la recuperación del estudio de una dimensión teórica y práctica innegablemente ligada a un mejor, por más profesional y vinculado al contexto, análisis de la educación y de la formación de educadores para ahora y para los próximos años.

No hace mucho, leyendo una obra sobre los contextos de la enseñanza en las escuelas secundarias (McLaughlin, 1990), subrayaba con firmeza quien suscribe algo de lo que allí se afirmaba, a saber, que la buena enseñanza, además del liderazgo y de las me- 
tas escolares, depende también de otro y más complejo conjunto de factores: de cómo piensan y sienten los profesores acerca de lo que hacen. No obstante, el gran peligro, como muy bien han advertido avezados analistas (ver Liston y Zeichner, 1987; Bullough, 1989; Smith, 1989) es el de que apelar a la reflexión se vaya convirtiendo -o se haya convertido ya- en algo similar a una especie de «slogan» educativo al que le falta suficiente elaboración conceptual y fuerza programática; o que, por eso mismo, y por otras causas, se convierta en un término sin mucho significado real y al margen de todo compromiso compartido de reforma y cambio en educación.

Para nosotros, el auge del énfasis en la noción de reflexión/reflexividad es el auge de la investigación cualitativa en el estudio de los procesos educativos, con una indisimulada carga de factores culturales y situacionales en su abordaje teórico. El acusado interés por el «pensamiento reflexivo» se debe igualmente, aquí y ahora, al progresivo reconocimiento de que la enseñanza supone un esfuerzo complejo, bastante específico de las situaciones y, en buena medida, dominado por dilemas que se vinculan a la filosofía, valores,... de cada profesor (dándose por sentado que el conocimiento profesional procede de fuentes, tanto externas como de las mismas interpretaciones que hacen los profesores de su experiencia diaria). De un modo u otro, es precisamente la primacía de la experiencia, y la noción de ésta como un genuino logro (Eisner, 1988), lo que se destaca sensiblemente en varias -y variadas- revisiones de trabajos focalizados en torno a la «práctica reflexiva» de los profesionales de la educación (cfr. Grimmet et al. 1990; Tom, 1985; Richardson, 1990; Wellington, 1991; Clift, Houston y Pugach, 1990). Hasta el punto de que no falta quien menciona la necesidad (McNamara, 1991) de fundir el conocimiento pedagógico formal -definido como el conocimiento pedagógico generado por la investigación sistemática y rigurosa- y el conocimiento pedagógico vernáculo (vernacular pedagogical knowledge) -definido como conocimiento pedagógico de trabajo desarrollado por los profesores a través de su experiencia- si se quiere que el estudio de la pedagogía contribuya de modo importante al desarrollo de una enseñanza más efectiva en nuestras aulas ordinarias.

\section{UN CAMBIO DE ACENTO TEÓRICO Y METODOLÓGICO}

Tal denominación de enseñanza o práctica reflexiva, con sus términos colaterales, y aún sinónimos, de «reflexión en la acción», «enseñanza basada en la indagación» y sus centraciones en el «profesor como tomador de decisiones» o el «profesor como solucionador de problemas» (...) implica al profesional, o al estudiante/candidato, en un ciclo de pensamiento y acción basado en la «experiencia», donde se representa a ese mismo «profesor» más como diseñador/artista que como un técnico/ingeniero, si bien tampoco se debe quedar uno con la idea, como luego veremos, de que en esa terminología no existen variaciones conceptuales referidas a la noción de reflexión abarcada. Normalmente, hay una derivación que lo deja todo más diáfano, esto es, el estudio del pensamiento reflexivo en educación privilegia una metodología de investigación/indagación narrativa (Connelly y Clandinin, 1990) en detrimento de la metodología (conductista) del positivismo lógico. Es la misma distancia explicitada por Calderhead (1987, p. 5; 1989, p. 43) cuando dice que los enfoques de enseñanza reflexiva en la formación y de- 
sarrollo profesional tienen que ver con la indagación crítica o la evaluación auto-dirigida, separándose de los enfoques de destrezas conductuales, parejamente llamados de «craft apprenticeship» (permítasenos la traducción de «aprendizaje artesanal»), en los que lo principal es la adquisición de destrezas predeterminadas de aula.

En oposición a la visión previamente imperante de la enseñanza como dominio (mastery) de una serie de conductas discretas/efectivas, se pretende una visión de los profesores como agentes activos en el desarrollo de su propia práctica, como sujetos que toman decisiones a la luz de un conocimiento especializado que guía sus acciones en situaciones concretas y que les anima a verse como miembros de una profesión compleja y desafiante. Pero quizás sea más ilustrativa la contraposición echando un ligero vistazo al movimiento centrado en el estudio empírico de la «teaching effectiveness» (para un estudio exploratorio de esta tendencia, ver, entre otros, Gage, 1986; Brophy y Good, 1986; Rosenshine y Stevens, 1986). Sabemos que la meta esencial de este «programa de investigación» ha sido identificar aquellas conductas y estrategias del profesional que tengan más posibilidades de conseguir resultados de logro en los estudiantes. Puesto que la búsqueda se ha ocupado de las relaciones genéricas -conductas docentes asociadas con logros académicos del alumno, dejando entre paréntesis otros aspectos- los hallazgos han sido relacionados más estrechamente con la forma de «dirigir» un aula que con las sutilidades de la intervención pedagógica efectuada (ver Ross y Kyle, 1987). Lo que querría decir que los principios de una enseñanza efectiva tendrían que ver, entre otros asuntos, con lugares donde los alumnos atienden sus tareas instructivas, o se orientan hacia el aprendizaje con un mínimo de disrupción/distracción; con el matiz adicional de que los propósitos educativos para los que esos resultados investigadores se hacen más relevantes apuntan hacia la enseñanza de destrezas. De ahí que Shulman (1987, p. 90) recoja muy oportunamente la observación de Rosenshine (1986) de que esta investigación tiene poco que ofrecer para la educación y la enseñanza de la comprensión (understanding), especialmente del material escrito complejo (mejor adecuación a la enseñanza de una destreza como la multiplicación que a la enseñanza de interpretaciones críticas de un texto. Se presenta, en una palabra, una perspectiva y una imagen de la enseñanza solidificada e institucionalizada según un modelo de «racionalidad técníca» (Clark y Yinger, 1987; Schon, 1983) en el que se sostiene que la práctica profesional destra es posible solo cuando el «practicante» emplea habilidades técnicas o de «ingeniería» basadas en el conocimiento sistemático, que es especializado, científico y estandarizado ${ }^{1}$. Y con el convencimiento absoluto de que «el práctico es un usuario del saber, un experto artesano que ejecuta el diseño del teórico» (Usher, 1991, p. 16). Baste esto, pues, como muestra de los graves inconvenientes de este modelo como paradigma válido de la educación, y sirva de precaución en la nada fácil construcción de una teoría de la educación epistemológica sólida (cfr. Touriñán, 1987).

1. El modelo de racionalidad técnica está perfectamente ejemplificado en el campo de la formación del profesorado con el auge de los modelos de formación basados en la actuación y/o competencia (P/CBTE, CBE son siglas evocadoras en la historia de las corrientes con influjo en la educación de los maestros -ver Santos Rego, 1985, 1986-) y los sistemas conductuales de evaluación de la eficacia docente. 


\section{SOBRE EL PROCESO DE REFLEXIÓN}

Concretar el sentido/significado del término «reflexión» no es nada sencillo, por más que se pueda acordar rápidamente que lo contrario de la acción reflexiva es la adesión automática/dogmática a principios o postulados que no se han sometido a examen ponderado. A un nivel muy amplio Ross (1989, p. 22; también Ross, 1990) se hace eco de la reflexión como una forma de pensamiento acerca de las materias educativas que supone la capacidad de hacer elecciones racionales y de asumir responsabilidad por ellas. Postura que coincide con algunos puntos de vista asentados en el campo (caso de los de Zeichner y Liston, 1987 o de Goodman, 1984) y en los que ya se delimitan algunos elementos del proceso de reflexión:

- reconocimiento de un dilema educativo.

- capacidad de respuesta a un dilema, sabiendo discernir tanto las similitudes con otras situaciones como las cualidades únicas que estas puedan presentar.

- enmarcamiento y re-enmarcamiento del dilema.

- experimentación con el dilema a fín de descubrir las consecuencias e implicaciones de las diferentes soluciones.

- examen de las consecuencias pretendidas y no pretendidas de una solución aplicada evaluando la deseabilidad de tales consecuencias.

En el ámbito de la formación de los profesores, lo que se ha escrito sobre la reflexión tiene casi siempre por epicentro de partida la famosa, aunque demasiado amplia, concepción de John Dewey al respecto:

«consideración activa, persistente y cuidadosa de cualquier creencia o forma supuesta de conocimiento a la luz de las bases que la apoyan y las posteriores conclusines a las que tiende» (Dewey, 1933, p. 9)

Así, la acción basada en la reflexión se consideraba como acción inteligente, en la cual se tenía presente su justificación y sus consecuencias (contrariamente a la acción ciega e impulsiva). Como afirma Calderhead (1989, p. 44), los rasgos de la reflexión que Dewey enfatizó eran el «sentido de asombro» ante un problema y la búsqueda propositiva y razonada de una solución. Sin embargo, para Armalin y Hoove (1989), lo que incorpora la idea del pedagogo de Chicago no es otra cosa que un seguro contra perspectivas rígidas y/o dogmáticas acerca de la educación/enseñanza/escolaridad, siendo un paso decisivo en el desarrollo de un antídoto contra la confianza de muchos docentes (por supuesto, sin plena conciencia de ello) en la mitología y la ficción. Estos mismos autores recuerdan también que la reflexión crítica no es un punto de vista, sino más bien un proceso dirigido a validar o invalidar un punto de vista dado. Porque, apelando de nuevo a este gran baluarte de la pedagogía en el siglo veinte:

«hay reflexión cuando empezamos a indagar la fiabilidad, la valía de cualquier indicación particular; cuando intentamos examinar su valor y ver qué garantía existe de que los datos disponibles apuntan realmente hacia la idea que es sugerida, de tal forma que se pueda justificar la aceptación de la misma» (Dewey, 1933, p. 11). 
Por eso, la no valoración del análisis crítico de la enseñanza cara a su justificabilidad puede convertir a las tentativas de estimulación de la reflexión crítica en los estudiantes y en los formadores de profesores en algo discutible y aún desprovisto de utilidad. El deseo de escrutar críticamente los fenómenos educativos refleja una voluntad dirigida a valorar la validez y conveniencia de políticas y prácticas escolares, junto con la disposición y aptitud para «cuestionar» la acción/nuestra acción como educadores y las asunciones fundamentales de tales acciones.

\section{AlgunOS ENFOQUES POSTERIORES A DEWEY}

Después de John Dewey se han sucedido, a tono con el vaivén de los tiempos y de las influencias de enfoques, planteamientos dispares acerca de la reflexión en la educación y en la enseñanza. Ahora bien, pocos han tenido el impacto y alcanzado la categoría de referencia segura en su estudio como los trabajos del norteamericano Donald Schon, primero con el título Reflective Practitioner: how professionals think in action (1983) y cuatro años más tarde con Educating the reflective practitioner. Tal vez el gran aliciente de su contribución sea el de haber ayudado a recuperar y mantener viva la tradición del conocimiento experiencial. Frente a las contínuas demandas de modos más tecnocráticos de funcionamiento en educación, se arguye que las propuestas de formas severas de «accountability» son totalmente equívocas. El problema, a su juicio, tiene que ver más con una crisis de confianza en las profesiones respecto de sus posibilidades de encontrar soluciones a las acuciantes cuestiones ambientales, sociales y económicas de nuestros tiempos. La base de su posición, tal como se ha expresado hace poco tiempo, es que:

«aquéllos que se empecinan en argumentar que la práctica profesional debería tener unas bases científicas incontestables y estar estrechamente unida a prescripciones extraidas de la investigación objetiva, iniciada desde el exterior y a gran escala, ignoran la medida en que el conocimiento derivado de la práctica es, de hecho, valioso y relevante en si mismo. Al centrarse exclusivamente en los productos de la investigación de otros, a expensas del proceso por el que se alcanza comprensión plena, lo que hacen los partidarios de tales perspectivas es tergiversar el valor de la investigación, el cual radica no en su ser definitivo, sino más bien en la provisionalidad (...), como algo a ser explorado, confirmado, o rechazado a la luz de la experiencia» (Smyth, 1989, p. 2; ver también Smyth, 1986, 1987).

Un punto interesante es aquel en el que Schon reclama atención preferente para el «planteamiento de problemas» en la práctica profesional, proceso que describe como una conversación reflexiva en la que el profesional recurre a su experiencia para entender la situación, enmarcando el problema, sugiriendo la acción y reinterpretando la situación a la vista de las consecuencias de la acción (Marsick, 1991). Con ello se ayuda a apuntalar una visión más equitativa del conocimiento como algo provisional y problemático, no un «a priori» instrumental, obligando casi necesariamente a meternos a fondo en la tarea de re-pensar la práctica profesional y, naturalmente, la relación entre teoría y práctica en la educación. Hoy, la mayoría de los profesionales (incluidos, por supuesto, los de la educación) se las tienen que ver con situaciones en las que las tareas a realizar 
no guardan mucha relación con aquellas para las que se supone que fueron preparados. En el sentido otorgado por Schon (1983, pp. 15-16. Tomado de Smyth, 1989, p. 3):

«las situaciones de la práctica no son problemas a resolver sino situaciones problemáticas que se caracterizan por la incertidumbre, el desorden y la indeterminación».

No sorprende, por tanto, que los profesores se sientan cada vez más inmersos en una dinámica de propósitos y valores en permanente conflicto.

La reflexión -para algunos deliberación- es, con todo, susceptible de ser conceptualizada según pautas y acentos diversos. En el lenguaje de Schon $(1983,1987)$ aparecen dos tipos: la reflexión-sobre-la-acción, esto es, reflexión sobre la práctica, una vez terminada una secuencia de esta, y sobre las propias acciones y pensamientos; y reflexiónen-la acción, o reflexión sobre los fenómenos y los modos espontáneos que tiene uno de actuar en medio de la acción. Se vincula al ejercicio de destrezas interactivas e interpretativas en el análisis y solución de problemas complejos y ambiguos.

Un tercer tipo de reflexión, la reflexión-para-la-acción, sería el resultado deseado de las dos anteriores. Reflexionamos, no tanto para revisar el pasado o para volvernos conscientes de los procesos metacognitivos que experienciamos, sino para guiar/orientar la acción futura. De ahí que la reflexión sea un proceso que abarca todos los «designios» temporales, pasados, presentes y futuros, simultáneamente. Y puesto que la educación no es una «hard science» necesitamos implicarnos en un desarrollo continuado del conocimiento para ampliar nuestra comprensión de los eventos escolares (Killion y Todnem, 1991). Desde luego, al examinar nuestras acciones pasadas y nuestras acciones del momento presente generamos conocimiento que a buen seguro habrá de informar nuestras acciones futuras. Lo vemos en el modelo de la figura 1, adaptado de Wildman et al. (1987) y tomado de Killion y Todnem (1991, p. 15), en el que se tratan de ilustrar los pasos que sigue la generación de conocimiento para la acción futura.

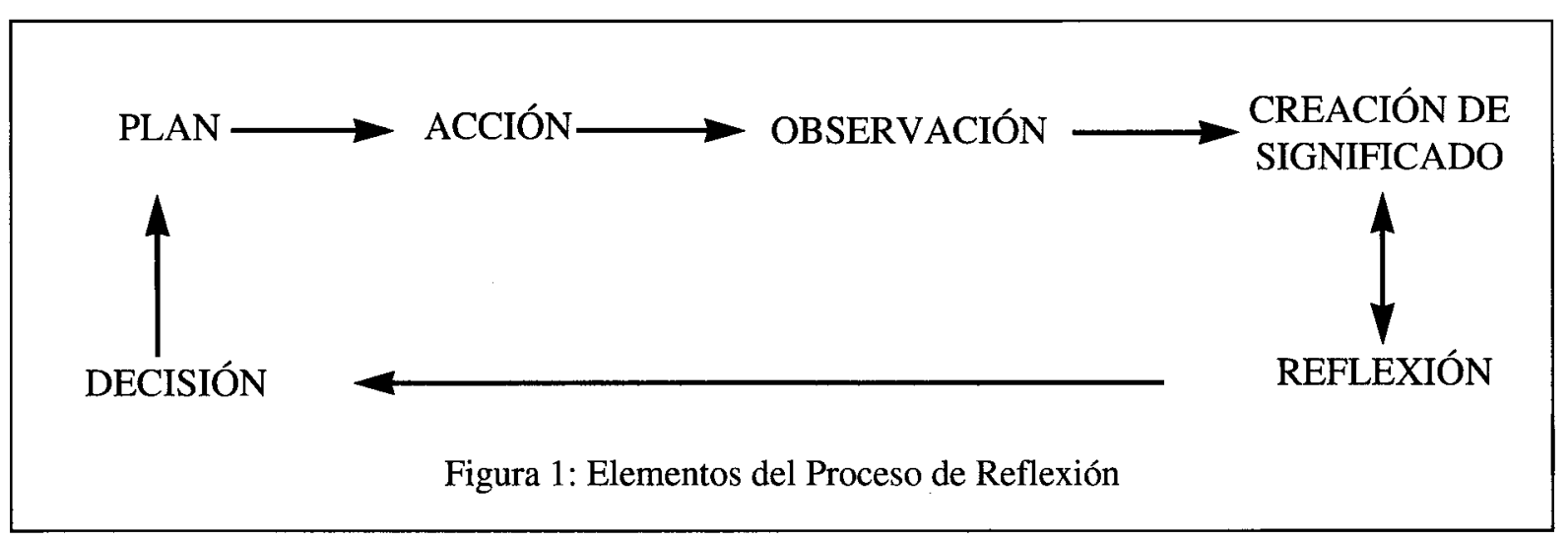

Muy sintéticamente, lo primero que hace el profesor es establecer un plan para la acción. Acto seguido, a través de la reflexión en la acción observa la acción tal como ocurre, casi como situándose al margen de la acción misma. Desde esta plataforma crea significado, al tiempo que comprende la dinámica de la relación causa-efecto que se da entre sus acciones y las respuestas consiguientes de los alumnos. Luego la reflexión sobre la acción y la reflexión para la acción impelen al profesor hacia el análisis de los eventos y la extracción de conclusiones que favorezcan su prospectiva decisional. 
Calderhead (1989) se hace eco de más posiciones/enfoques que han venido influyendo sobre este 're-surgir' de la educación reflexiva, destacando las correspondientes a filósofos de la acción práctica (Gauthier, 1963; Polanyi, 1967) y teóricos del curriculum como Scwab (1971) y Van Manen (1977). Es notorio el punto de vista de Gauthier (1963): ser capaz de establecer razones para la acción, dice, deliberar de acuerdo con esas razones y actuar según la propia deliberación son aspectos de la acción autónoma y responsable.

De otra parte estaría la acción prestada a la que llamamos en ciencias sociales «Escuela de Frankfurt», particularmente a través de la obra de J. Habermas, auténtico inspirador de un concepto de reflexión como auto-determinación/emancipación ${ }^{2}$; es el proceso de hacerse consciente del propio contexto, de la influencia de las presiones sociales e ideológicas sobre las prácticas previamente asumidas, y de la consecución del control sobre la dirección de esas influencias (cfr. Habermas, 1982, 1984; Radl, 1991). Se trata del concepto usado en los últimos tiempos para respaldar un 'paradigma' de investigación-acción extendible y extendido hacia el de «maestro como investigador» (Carr y Kemmis, 1988; Hopkins, 1989; Elliot, 1990) en el que se afirma la conveniencia de que los profesores logren una mejor 'auto-determinación profesional' por medio de la comprensión y el conocimiento en profundidad que acompaña a la investigación/indagación cuyo foco único es su situación.

\section{REFLEXIVIDAD Y FORMACIÓN DEL PROFESORADO}

Viendo la literatura existente sobre el tema y leyendo algunos de los estudios más citados uno se percata rápidamente de la notable varianza en el modo de justificar, defender y relacionar los conceptos en la educación profesional de los profesores. Ya se ha podido intuir que la oscilación en el soporte de justificación va desde el énfasis puesto en la 'responsabilidad moral' al puesto en la 'efectividad técnica'; así se entiende que la noción de 'reflexividad' se haya ido incorporando a cursos de acción tan divergentes en la formación del profesorado como los que emplean sin reparo alguno un enfoque de destrezas conductuales (afianzando la ya clásica habilidad de muchos conductistas para reconceptualizar adaptativamente sus antiguas proposiciones) -la 'reflexión' no sería más que un medio para el logro de ciertas prácticas prescritas-, y los que se alinean al lado de un enfoque de teoría/ciencia crítica (critical science approach), para los que el objeto de la reflexión no es otro que la emancipación y la autonomía profesional. De hecho, lo que pasa en este complejo asunto es que los investigadores y los educadores de maestros, profesores, pedagogos, etc. conectan sus propias nociones de lo que es o puede ser reflexión con su más o menos dilatada gama de creencias acerca de la educación, de la enseñanza y de la preparación de sus profesionales (cfr. Calderhead, 1989).

Asimismo, para resumir, es evidente que la conceptualización de la 'reflexión'/reflectividad', audible en debates y reuniones de personas interesadas, no es la misma ya

2. A través de Habermas, o por la repercusión de sus postulados, se ha derivado una noción de «pedagogía emancipatoria», bien analizada y presentada por Brezinka (1990) como una pedagogía no científica sino ideológica, una teoría práctica y no una teoría científica de la educación, amén de estar cargada de indicaciones extremadamente vagas. 
que, para unos, lo que importa sobremanera es el proceso de la reflexión (reflexión en la acción, sobre la acción, ...), para otros es el contenido (por ejemplo, los valores que presiden la vida de los docentes, el contexto social inmediato, la teoría educativa que les anima, los patterns de acción en el medio educativo, etc.), mientras que otros, preocupándose naturalmente de proceso y contenido, atienden también a las precondiciones (las actitudes hacia la reflexión, el contexto en el que se da,...) y al producto de la reflexión (niveles de eficacia y/o rendimiento, emancipación, comprensión de la relación entre valores y práctica,...). Esta varianza de puntos de vista podría remitir, con idéntico propósito, al marco de Tom (1985), que clasifica lo relativo a la reflexión en tres dimensiones: de la problematicidad implícita, de la indagación (inquiry) y del status ontológico de los fenómenos educativos. Y sin dejar en el tintero medios adicionales/alternativos que todos conocemos para discriminar los conceptos de reflexión: la relación entre teoría y práctica, pensamiento y acción, fines y medios, etc.

Ahora, pensando fundamentalmente en los ángulos y direcciones que fue tomando la investigación acerca del pensamiento reflexivo de los profesores, intentaremos delimitar algunos de tales ángulos y elementos que vienen conformando un devenir investigador, que no es nada monocorde en sus propuestas, ni está exento de encendidas polémicas en las conclusiones sobre temas principales. Parte de esta intrincada tarea de ordenación y recapitulación ha sido realizada en los últimos tiempos (ver Simmons et. al., 1989; Sparks-Langer et. al., 1990; Sparks-Langer y Bernstein, 1991) y de esos esfuerzos partimos, con la intención de integrar y ajustar, pedagógicamente hablando, lo que nos parece más sustancial en sus derivaciones, comentarios y conclusiones.

\section{REFLEXIÓN Y ACCIÓN DEL PROFESOR: EL EJE COGNITIVO}

En general, y sin que ello suponga una anotación exhaustiva, puede decirse que los elementos cognitivos de la reflexión se centran en cómo usan los profesionales el conocimiento en su planificación y toma de decisiones. Fijémonos, por su importancia, en la categorización realizada por un destacado pedagogo de la Stanford University, Lee Shulman, hace cuatro años. Decía entonces este Profesor que si tuviéramos que organizar el conocimiento del docente en un manual tendríamos que incluir, al menos, estas categorías:

1) conocimiento de contenidos disciplinares.

2) conocimiento pedagógico general: con atención explícita a los principios y estrategiạs de intervención (vinculados al aprendizaje y motivación educativas, gestión y organización de la educación, etc.) que transcienden a una materia concreta.

3) conocimiento del curriculum, haciendo hincapié en el 'control' de materiales y programas idóneos.

4) conocimiento de contenido pedagógico, que viene dado por esa especie de amalgama entre contenido y pedagogía, definidora, en sentido amplio, del conocimiento profesional de los mejores profesores.

5) conocimiento del alumno y sus características.

6) conocimiento de los contextos educativos, desde los contextos de aula a los propiamente comunitarios y socio-culturales. 
7) conocimiento de los fines, propósitos, y valores educativos, junto con sus raices histórico-filosóficas.

De las mencionadas categorías, Shulman (1987) destaca el conocimiento de contenido pedagógico como más relevante porque identifica «cuerpos de conocimiento» necesarios en la enseñanza y representa, sigue afirmando, la mezcla de contenido disciplinar y pedagogía dentro de un marco comprensivo vinculado a la forma en que temas y problemas son organizados, representados y adaptados a los diversos intereses y aptitudes de los alumnos. No obstante, el auténtico problema sigue consistiendo en averiguar de qué modo se relacionan entre si todas estas clases de conocimiento, configuradoras, al fin y a la postre, de un conocimiento profesional base en educación ${ }^{3}$. Así lo reconocen Wilson, Shulman y Richert (1987, p. 118) antes de emplazarse a sí mismos y a los demás investigadores para proseguir el trabajo por esta vía.

Pero puesto que la enseñanza tiene que preocuparse por los medios y por los fines, hay procesos de razonamiento que subyacen a ambos. Una meta de la formación de profesores consiste en prepararles para un razonamiento sólido (Fenstermacher, 1986) que exigirá un proceso de pensamiento sobre lo que hacen, así como una adecuada base de hechos, principios y experiencias desde las que poder razonar con suficiente rigor. Los estudios de Wilson, Shulman y Richert, y de Shulman en solitario, ambos publicados en 1987, coinciden al cien por cien no sólo en realzar los procesos de acción y razonamiento pedagógico, sino también en la mención de los mismos aspectos en tales procesos, los cuales -considerados desde el punto de vista del profesor-suponen un ciclo que empieza y termina con un proceso de comprensión (aunque de naturaleza distinta), pasando por los de transformación, instrucción, evaluación, y reflexión (ver Figura 2).

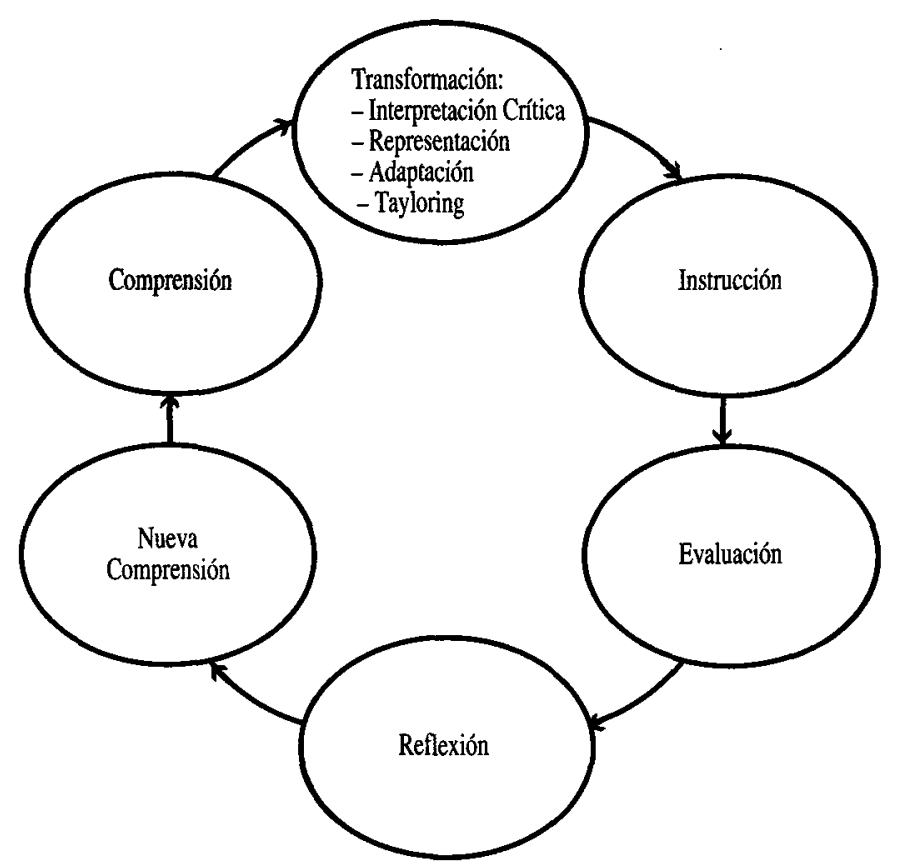

Figura 2: Modelo de razonamiento pedagógico (Tomado de Wilson, Shulman y Richert, 1987, p. 119)

3. «Pero la clave para distinguir el conocimiento base de la enseñanza radica en la intersección de contenido y pedagogía, en la capacidad de un maestro para transformar el conocimiento de un contenido concreto que posea en formas pedagógicamente significativas y valiosas, y adaptadas a las diferencias que presentan los alumnos» (Shulman, 1987, p. 15). 
Al principio está un proceso de comprensión: los profesores deben entender críticamente un conjunto (set) de ideas y contenidos, en términos de su estructura sustantiva y sintáctica; pero deben comprender también las relaciones entre ese contenido en cuestión y otras ideas dentro de ese contenido e ideas en dominios próximos.

El proceso de transformación implica cuatro subprocesos que se combinan o unen en un set de estrategias de intervención: uno de interpretación crítica (analizar/revisar los materiales a la luz del conocimiento que uno tiene de la materia), otro de representación (formas alternativas que tiene un profesional de representar una materia, un tema o una lección, siendo de gran utilidad la posesión de un repertorio de metáforas, analogías, ilustraciones y ejemplos porque facilitan enormemente la tarea), otro más de adaptación general (ajuste de la transformación a las características de los estudiantes en general, sin perder de vista sus patrones motivacionales) y, por fin, uno de adaptación específica (tayloring), esto es, de acompasar el material con los alumnos concretos de una clase más que con los alumnos en general.

La instrucción tiene que ver con la actuación observable del profesor e incluye los rasgos/características de una intervención efectiva (organización, agrupamientos, previsión de ritmos, coordinación de actividades de aprendizaje, explicación, etc.). El proceso de evaluación se da durante y después de la instrucción, en la medida en que los profesionales «controlan» los niveles de comprensión alcanzados por sus alumnos. Mientras tanto evalúan su propia enseñanza y modos de intervención a través del proceso de reflexión, aquí y ahora ligado al aprendizaje que parte de la experiencia y que permite una reconstrucción de eventos, emociones y realizaciones. Y cerrando el círculo llegamos a donde empezamos, si bien alcanzando una «nueva comprensión», estimulada sin duda por el mayor conocimiento alcanzado respecto de los propósitos instructivos, el contenido temático/disciplinar y/o la metodología más apropiada.

Volviendo a las categorías de ordenación del conocimiento base, se ha detectado un vacío investigador sobre la percepción docente acerca de categorías como pueden ser el 'conocimiento de los contextos educativos' y el conocimiento de propósitos, fines y valores, limitándose muchas veces los estudios a lo que Van Manen (1977) ha llamado el nivel técnico de la reflexión, donde la cuestión predominante es la aplicación eficiente y efectiva del conocimiento educativo, con los propósitos de alcanzar fines que son aceptados como algo dado, pero donde, sin embargo, ni los fines ni los contextos institucionales de aula, escuela, comunidad y sociedad son tratados a la vista de su problematici$\mathrm{dad}^{4}$.

4. Este mismo investigador ha demostrado una buena sagacidad conceptual al identificar otros dos tipos/niveles de reflexión/reflexividad: el primero -que sería el segundo en su propuesta- está basado en una concepción de la acción práctica que remite el problema a la explicación y clarificación de las asunciones y predisposiciones subyacentes a los «practical affairs» y a la evaluación de las consecuencias educativas que conlleva una acción (lo que se considera en este nivel es que toda acción está unida a planteamientos/compromisos valorativos concretos); y el segundo, nivel de reflexión crítica, que incorpora criterios morales y éticos en el discurso sobre la acción práctica. Las preguntas centrales girarían alrededor de las metas, experiencias y actividades educativas que llevan hacia formas de vida mediadas por preocupaciones/inquietudes de justicia, equidad, etc. incluyendo también el tema de si las actuales perspectivas/configuraciones sirven a importantes necesidades y satisfacen igualmente no menos importantes propósitos humanos (cfr. Zeichner y Liston, 1987; Tom, 1985). 
Sobre lo que se han volcado mayores esfuerzos, siguiendo con el mismo foco cognitivo, ha sido sobre la organización del conocimiento base. Al respecto, es preciso anotar con rapidez que, bajo ángulos de visión cognitivista, la información se organiza en un «network» de hechos, conceptos, generalizaciones y experiencias relacionadas; justamente es así como hablamos de esquemas a modo de estructuras que permiten la comprensión del mundo por el sujeto, el almacenamiento de la información y, lo que es clave, un acceso rápido a la misma (cfr. Berliner, 1986).

Curiosamente, la noción de esquema ('schema'), intercambiable cuando se habla de estructuras de conocimiento por la de «marcos» (frames), «guiones», «prototipos», etc. (ver Nisbett y Ross, 1980), ha sido profusamente empleada en compilaciones y revisiones investigadoras que intentan poner de manifiesto diferencias potenciales entre profesionales nuevos/novatos/advenedizos/'faltos de rodaje' y profesionales expertos/con experiencia, para indicar -pongamos por caso- que mientras los últimos cuentan con esquemas más profundos y con más riqueza de conexiones para extraer información a la hora de tomar decisiones, los primeros parecen tener esquemas más 'débiles' y menos desarrollados. Lo que se corresponde, de modo bastante lógico por cierto, con hallazgostipo (ver Calderhead, 1983; Clark y Peterson, 1986; Leinhardt y Greeno, 1986) apuntando a que los 'esquemas' de los profesores en relación con sus alumnos pueden diferir marcadamente de los esquemas de que pueden hacer gala sus colegas recién llegados o con pocos años de experiencia docente. Cuestión vidriosa es la de si sería posible enseñar a los «recién llegados» o a los aspirantes las estructuras de conocimiento que usan los más expertos (cuidando de no unir sin más «persona con experiencia» y «persona experta» en educación). Y es que bastantes estudios cognitivos no muy alejados de nuestro tiempo comenzaron a definir esa «pericia educativa» desde la perspectiva del uso del conocimiento en la práctica, arguyendo que la adquisición y uso del conocimiento experto está más condicionado por los contextos particulares de lo que nos parecía antes. La idea de que tal «expertise» es contextual -lo decimos con Lampert y Clark (1990)- penetró las teorías sobre procesos cognitivos en educación a medida que se fue describiendo el conocimiento no formal de practicantes «poco escolarizados» pero altamente diestros en varios dominios/ámbitos, concurrentemente al examen de ciertas teorías sociales acerca de la relación entre el lenguaje y el desarrollo del pensamiento (el hecho, incluso, de que la mayor parte de la actividad escolar se da en una cultura representa un punto no marginable cuando se aspira a comprender muchas de las dificultades de aprendizaje en la escuela -ver también Lave, 1988-).

En torno a esto, lo que aparece de nuevo como preocupante es que se haga equivalente la afirmación del contexto con el visto bueno a un relativismo contextual, acompañado de un pesimismo sistemático acerca de la utilidad de cualquier investigación del pensamiento docente para la formación de los profesores. Tal es el núcleo de la divergencia entre Floden y Klinzing (1990) y la opinión de Clark y Lampert (1986), Clark (1988) y Lampert y Clark (1990). Defender, como hacen estos últimos en su artículo de hace un lustro (1986), que los profesores deben ser el único juez de su enseñanza, que lo que hay que darles son instrumentos conceptuales para comprender cada situación educativa concreta, y que la investigación solo puede plantear preguntas porque son los maestros y sus formadores los que han de proporcionar las respuestas, es en la perspectiva de aquéllos (Floden y Klinzing, 1990, p. 16) tanto como alentar abiertamente una posición que hace de la absoluta confianza en la experiencia personal el primer baluarte de 
la enseñanza. La investigación, continúan, puede ser fuente de recomendaciones generales para la acción, «recomendaciones que son respuestas y no simples preguntas, proporcionando una dirección susceptible de ser considerada reflexivamente, no mero alimento para el pensamiento. Y con el poder de sugerir cambios sustantivos en la práctica de la formación de los profesores, lejos de razones palmariamente favorables al mantenimiento del «status quo» (p. 17). Para no alargarnos, si edificante puede resultar un camino intermedio entre la prescripción radical y la racionalización del «status quo», lo propio cabe decir de la propuesta de Lampert y Clark (1990) pidiendo que en la «teacher education» se tenga en cuenta no sólo el contenido del pensamiento de los «profesores expertos» sino también las condiciones bajo las cuales piensan y usan ese conocimiento.

Con todo, los argumentos acerca de la existencia de esquemas y los que se basan en la naturaleza situada de la cognición son intentos teóricos de explicar la relación entre conocimiento y pericia (expertise). La teoría de la cognición situada, fundamentalmente asociada al reflujo de los postulados vygotskianos en educación (cfr. Rogoff y Lave, 1984; Rogoff, 1990; Suchman, 1987) defiende que todo conocimiento es una construcción conjunta de la mente y de la situación en la cual la mente tiene que hacer frente a un problema. Hoy en día, quizá su gran valor sea el de que ayuda a explicar mejor el «buen hacer» en la intervención educativa/pedagógica del profesional que la teoría de las estructuras de conocimiento/schemata theory, de configuración más abstracta. Así pues, aceptando que el aprendizaje y la cognición son/están fundamentalmente situados contribuiremos a inaugurar alrededor nuestro una cultura del aprendizaje más significativa y tan necesaria en la actual dinámica de reforma educativa, además de abrir vías para una nueva epistemología vinculable a la mejora en el aprendizaje (estructurándolo según presupuestos colaborativos) y a nuevas perspectivas en educación. La actividad en la que el conocimiento se desarrolla y se despliega -esgrimen Brown, Collins y Duguid (1989) - no se puede separar del aprendizaje y la cognición. Ni es neutral. Es, más bien, una parte integral de lo que es aprendido. Dando derecho a decir que las situaciones coproducen el conocimiento a través de la actividad ${ }^{5}$. En una palabra, ignorar la naturaleza situada de la cognición puede tener la consecuencia de frustrar la misma meta educativa de procurar un conocimiento robusto y aprovechable.

En lo que se han hecho apreciaciones convergentes ha sido en la cuestión de la «automaticidad» como elemento notorio en el pensamiento de los «expertos»: ciertas rutinas (secuencias de respuestas) son estimuladas directa/automáticamente por una situación y puestas en acción con poca atención consciente por el profesional, llevándole a ejecutar ciertos comportamientos de modo inconsciente mientras atiende sin dificultades a aquellos eventos más novedosos o que tienen en ese momento o coyuntura más prevalencia para Ella o para El. No serían, pues, sino esquemas concebibles cual «guiones» facilitadores directos de la acción. Claro está que esto no se produce automáticamente en sentido absoluto sino que también cuenta la experiencia y sus efectos en el procesamiento del profesor. Los teóricos constructivistas -huelga citar los clásicos trabajos de

5. Conviene reparar en la frecuencia con la que, al hablar de aprendizaje en esta literatura, se emplea, además del término «learning», el de «apprenticeship», término estrechamente unido a nuestra imagen del conocimiento como instrumento; y que ayuda a enfatizar, como bien refieren Brown, Collins y Duguid (1989, p. 39) la centralidad de la actividad en el aprendizaje y el conocimiento, ilustrando la naturaleza enculturizada, situada e inherentemente contextual de ese mismo aprendizaje. 
Piaget- lo han planteado con atinada perspicacia: estamos creando constantemente nuestros propios significados; lo que llama a un proceso dual de asimilación (adaptación de lo nuevo con lo viejo) y acomodación (cambio de la vieja organización mental para incorporar la nueva), contribuyendo, por otra parte, a realzar la importancia e influencia de los filtros culturales (valores, creencias,...) en la percepción e interpretaciones de informaciones. No es raro, entonces, que en la educación de los maestros se atienda con cierto esmero (ver Ross, 1990) al análisis del potencial nexo entre preconcepciones referidas a fines y metas de la educación y aprendizaje en los programas de preparación.

Otro aspecto del eje cognitivo al que se podría dedicar mucho más espacio del que aquí es posible es el de la metacognición ${ }^{6}$ de los profesores y en el que se podría revisar la presencia y efectos de sus percepciones de auto-eficacia, de su proceso (y subprocesos) de aprendizaje autoregulado, conductas y acciones propositivas, etc. El profesor «reflexivo» adquiere la capacidad de controlar el efecto de las acciones que ha emprendido, junto con los procesos cognitivos que median en su particular toma de decisiones, implicando estos la elaboración de inferencias y, por supuesto, de hipótesis provisionales. Obviamente, una vez más, también este ha sido y sigue siendo terreno abonado para una previsible confirmación de que son los más «expertos» los que exhiben conductas, acciones y aprendizajes más auto-regulados que los que, por así anotarlo, están o creen estar en camino de serlo.

Al margen de los puntos representativos en la investigación no está de más reparar en alguna propuesta de acción en el campo de la preparación de docentes, pensada y articulada hacia la promoción de la reflexión cognitiva. Como, por ejemplo, la realizada en Michigan (EE.UU.) bajo las siglas C.I.T.E./«Collaboration for the Improvement of Teacher Education» («Colaboración para la Mejora de la Educación de los Profesores») y formando parte de un programa para no graduados de cuatro años de duración. Según sus diseñadores y propulsores (Sparks-Langer et. al. 1990; Sparks-Langer y Bernstein, 1991; Pasch et. al., 1990), partiendo de experiencias de campo estructuradas, algunas semanas de enseñanza real en aulas, asignaciones escritas, microenseñanza, etc. se consiguió ayudar a los estudiantes a analizar y reflexionar acerca de los temas presentados en los cursos. Sin embargo, lo mejor fue el descubrimiento de un marco para la evaluación del pensamiento reflexivo. Tomando como referente lo manifestado en entrevistas centradas en determinados eventos educativos se llegaron a delimitar niveles del siguiente tipo: no descripción, descripción simple, etiquetación de sucesos empleando lenguaje pedagógico, explicación basada en la tradición o en un enfoque idiosincrático, explicación que hace uso de principios de acción pedagógica, explicación que se basa en principios pedagógicos y características del contexto, y explicación que incluye consideraciones ético-morales. Si el lector vuelve a leer estos niveles y los toma en sentido progresivo se percatará de la sucesiva implicación que suponen de esquemas cada vez más sofisticados en los profesionales de la educación.

Siguiendo coordenadas próximas, Grinberg (1989) realizó un contraste de estudiantes en el programa CITE con un grupo que nada tenía que ver con esta iniciativa de entrenamiento docente. Bueno, pues aunque ambos grupos eran inicialmente iguales en las

6. Aquí aceptamos la alusión a lo metacognitivo hecha por Zimmerman (1989): procesos de toma de decisiones que regulan la selección y uso de varias formas de conocimiento. 
'puntuaciones' de pensamiento reflexivo y en otros factores, los candidatos inmersos en la tentativa colaborativa consiguieron valoraciones superiores en su pensamiento reflexivo. ¿Qué resultaba?. Pues que, al menos aparentemente, los cursos que incluían experiencias de campo guiadas promovían mayor reflexión que los carentes de esas experiencias.

\section{EL ENFOQUE CRÍTICO: OTRO EPICENTRO DE LA REFLEXIVIDAD}

Empecemos (re)marcando una diferencia: el enfoque crítico de la 'reflexión' debe su nombre a la fijación que supone, junto a medios y fines, en los aspectos morales y éticos de las opciones y decisiones educativas y sociales en las que se enmarca la vida de los profesionales. Recuérdese que el énfasis del apartado anterior se ponía en el 'cómo' de la toma de decisiones. En este se privilegia la sustancia que dirige/orienta el pensamiento (experiencias, creencias, valores socio-políticos, metas/aspiraciones de los profesores,...). Para entenderlo bien, permítasenos reiterar que los elementos críticos de la reflexión se oponen, con bastante asiduidad, a los de la reflexión técnica, empleando la división de Van Manen (1977), y donde al profesor le ocupa sobre todo la búsqueda de medios para el logro de un fin no siempre suficientemente examinado (sería el caso de una re-configuración de los espacios educativos con el único propósito de mantener el control por el control mismo, omitiendo cualquier consideración de otros efectos posibles). En definitiva, por esa vía se incurre no pocas veces en un notorio olvido, que en ocasiones llega a la marginación consciente, de estructuras interactivas y de aprendizaje cooperativo en la educación y en la formación de los profesores, con lo que la propia práctica discurre vacía de resortes experienciales válidos para una mejora efectiva de la enseñanza (cfr. Santos Rego, 1989, 1990).

Decir 'acción reflexiva' y 'teoría crítica' es señalar un par de conceptos unidos a la comprensión de la reflexión crítica en educación. Explayarse un poco sobre el primero de ellos exige retomar la sobresaliente aportación de Schon $(1983,1987)$, por ser el primero en analizar a fondo el trabajo de los profesionales. Algo con lo que se encontró, sorprendiéndose y sorprendiendo, fue el poco énfasis que ponen determinados profesionales en los modos tradicionales de resolución de problemas. Brevemente, más que implicarse en un proceso racional de selección de la mejor solución hacia una meta de interés común, muchos profesionales inician y desarrollan un debate en el que caben, entre otros, la naturaleza de las decisiones, el valor de las metas o las consecuencias últimas de las acciones; diálogo reflexivo que refinó como «planteamiento de problemas» (problem setting). Igualmente, si bien no se cuestiona en absoluto la adquisición de conocimiento profesional a través de principios y destrezas educativas aprendidas/adquiridas, se afirma que el grueso del aprendizaje de esos profesores procede de la permanente dialéctica acción-reflexión en contacto con los problemas cotidianos. Con lo cual solo resta añadir que gran parte de la información lograda a través de la experiencia es tácita y que no es un conocimiento cognitivo básico para enseñar lo que va forjándose sino, más bien, un sistema de apreciación global que deja su huella en la definición de situaciones, en la advertencia y realce de hechos, en los interrogantes generados, o en las decisiones que toman los profesores. 
Nos queda la mención más generosa de los que avalan una referencia a la reflexión en términos de Teoría Crítica. Su postura, radicalmente militante, se podría hermanar con lo que McLaren (1989), por ejemplo, observaba para lo que el mismo bautizó como «Pedagogía Crítica», cuya misión no ha de ser otra que proporcionar a los maestros/pedagogos e investigadores más y mejores medios de entender el rol de la escuela en una sociedad que responde a contrapuestos intereses de clase, etnia, sexo, etc. Para ellos, lo destacaba implícitamente Smyth (1989, p. 3. Ver, asimismo, Smyth, 1987), el principal impedimento que se divisa, al tiempo que el principal desafío, en el enfoque reflexivo se sitúa en las prolíficas tentativas de reforma que responsabilizan directamente a la escuela de las necesidades económicas fuera de ellas; lo cual es santificar la mitología de que, en el fondo, las escuelas y los maestros son la causa de la situación económica, por lo que no se puede prorrogar más la vuelta a la enseñanza de destrezas básicas, la implantación de sistemas de evaluación docente y de normas disciplinares firmes,... en pos de una restaurada visión de la escuela supeditada a los objetivos de la economía.

Contra ese estado de cosas y a modo de enseña para el combate emplean la noción de «empowerment» («fortalecimiento») con la intención de criticar y descubrir las tensiones existentes entre las prácticas de una/o y los contextos socio-culturales en los que nos movemos. Promoviendo un cambio en la correlación de fuerzas a medida que los practicantes abarcan más posibilidades de acción. Tal «fortalecimiento» a través de la reflexión lo expresaba Simón (1987, p. 374. Citado en Smyth, 1989, p. 5) sentenciando:

«lo que literalmente significa es conseguir aptitud para, permitir o facilitar. Cuando escuchamos la palabra empowerment en educación, normalmente tiene un sentido de crítica. Su referente es la identificación de las relaciones opresivas e injustas que limitan el pensamiento, los sentimientos y la acción humana. Limitación que impide a la persona participar en igualdad de condiciones con otros miembros del grupo o de la comunidad (...). To empower es permitir hablar a los que han sido silenciados».

Por tanto, la pretensión última es avivar un discurso sobre el papel de las escuelas en la sociedad democrática. Se necesita entonces ayudar a los profesores a clarificar sus propias creencias acerca de los propósitos de la educación y a examinar en profundidad métodos y materiales, en un proceso de aprendizaje de lecciones ocultas que también contiene la misma realidad aparente de la escuela. No hay duda de que para ellos el conocimiento es construido social y simbólicamente a través de la interacción y la relación con los demás. Y si inquiriéramos diferencia con el constructivismo cognitivo no nos quedaría más remedio que reparar en el mayor acento que pone en los conceptos de moral, justicia, democracia y solidaridad.

Dentro del punto de vista que representan los así llamados «teóricos críticos», la convención esencial es que a medida que los profesores describen, analizan y hacen inferencias acerca de los eventos que suceden en el aula, lo que hacen es crear sus propios «principios pedagógicos», incluso teorías a pequeña escala, o de corto alcance, que dan sentido a lo que ocurre y guían las acciones subsiguientes. Al inicio de este trabajo mencionamos cinco componentes del pensamiento reflexivo identificados por Ross (1989, 1990). Pues bien, quien se detenga un instante en los tres primeros seguramente podrá ver la proximidad al proceso que Schon rotuló como «Problem Framing». Y si la atención continúa estará en condiciones de advertir que el cuarto y el quinto de aquellos elementos conducen en última instancia a un proceso de pensamiento clave en la «Pedago- 
gía Crítica», nada menos que el de la relación entre el poder y el conocimiento, con el aserto anexo de que ese mismo conocimiento ha de valorarse según la forma que tenga de justificar o marginar visiones concretas del mundo.

De otra parte, también en el ámbito universitario se ha considerado la meta de la reflexión crítica en programas de formación, aunque su alcance/promoción efectiva no se presente tan fácil como en los casos de la reflexión técnica o de la reflexión práctica. Permítasenos volver un momento a un trabajo de Ross (1989). En la evaluación de los efectos de un curso (la investigación en la educación primaria) que formaba parte de un programa de preparación de profesores (genéricamente llamado «Proteach») se pensó en estimular la reflexión ayudando a los estudiantes a examinar sus propias creencias, socialmente construidas, acerca de las escuelas y la enseñanza. Se requerían proyectos de investigación-acción junto con trabajos de los propios estudiantes centrados en la transición «de la teoría a la práctica». Así, con el propósito de evaluar el pensamiento de los alumnos esta profesora y sus colaboradores asignaron un «nivel de reflexión» a cada uno de los 134 «theory-to-practice papers», desde 1 (bajo: descripción con poco análisis de contexto) a 3 (alto: multiplicidad de perspectivas con reconocimiento de gran impacto de las acciones de los profesores), si bien una gran parte de los trabajos (papers) fueron calificados dentro de los niveles 1 o 2 (niveles «bajo» y «moderado»). Este y otros estudios del programa citado les fueron convenciendo de que la base del desarrollo de la práctica reflexiva residen en lo que llaman «cambio en perspectiva». Conclusión: tratemos de que los profesores que ya lo son y los que se están preparando para serlo construyan sus propias perspectivas aprovechando sus experiencias personales y profesionales, pasadas y presentes, en las escuelas; considerando su conocimiento teórico general, sus percepciones de auto-eficacia y sus interacciones con profesores, iguales, supervisores y alumnos en la «institución».

Si volviéramos la vista, por tanto, hacia lo que han sido y son estos programas, tal vez no podríamos sustraernos al hecho de su limitado éxito en la promoción de la reflexión crítica. Lo que compensan con su apreciable contribución en forma de 'marcos' y técnicas favorecedoras de una mejor descripción del pensamiento reflexivo en el medio escolar (técnicas/procedimientos incluibles serían: escritura de diálogos estructurados, examen de perspectivas múltiples, diálogo crítico, estudio de experiencias de campo, investigación-acción, etc.) y de una afirmación de la sustantividad y enjundia de los métodos cualitativos e interpretativos para avanzar en el conocimiento de lo que pasa en los escenarios educativos. Ningún engarce mejor para aproximarnos ahora a las 'narraciones' y 'relatos' como fuente y base de tal conocimiento.

\section{EL TERCER ESLABÓN DE LA REFLEXIVIDAD: LA SUSTANCIA DE LOS RELATOS}

Indudablemente, la indagación narrativa ha experimentado un creciente auge en los estudios acerca de la experiencia educativa. Una razón esgrimida con bastante tino para el uso de la narración en la investigación educativa es que los humanos somos organismos que contamos relatos (storytelling organisms). En sentido global, estudiar las narraciones es estudiar las formas en que experienciamos el mundo, lo cual se traduce en una visión de que la educación no es más que la construcción y reconstrucción de relatos 
personales y sociales; esto es, ¿qué son los profesores y los alumnos sino contadores de historias y personajes de las suyas propias y de las de los demás (Egan, 1986; Santos Rego, 1990a). En la revisión de la literatura realizada por Sutton-Smith (1986) se distinguía entre enfoques estructuralistas, que se basan en el concepto de 'schema' y otros en idéntica dirección cognitiva, y enfoques de significado en una tradición hermenéutica. Esa literatura ha sido bautizada en el curriculum mediante la sugerencia, pedagógicamente sagaz, de que se proceda a organizar la materia/disciplina en forma de buen relato (ver Egan, 1991, pp. 202-218), sin pensar exclusivamente en el alcance de objetivos específicos. En esto, haciendo caso de lo que escribió Jackson (1987, p. 307), sería factible sostener que «aún cuando la materia de estudio no es en si misma un relato, sus contenidos contienen normalmente un determinado número de segmentos narrativos».

Es comprensible que, estando su centro en la experiencia y en las cualidades de la vida y de la educación, haya que situar a la narración en una matriz de la investigación cualitativa en pedagogía, aunque sus manifestaciones se extiendan lógicamente más allá de nuestro campo principal de estudio (psicología, antropología, sociología,...). En lo que nos es más próximo, la esencia de la parte narrativa de la reflexión se podría desprender sin mucho problema de las siguientes palabras: «lo que se desconoce en el conocimiento base de la enseñanza son las voces de los mismos profesores, las preguntas que estos hacen, los modos que estos tienen de usar la escritura y el habla intencional en sus vidas laborales y profesionales, y los marcos interpretativos que emplean los profesores para comprender y mejorar sus propias prácticas escolares» (Cochran-Smith y Lytle, 1990, p. 2). Así, por más que la 'narrativa' del profesional de la educación pueda integrar dimensiones cognitivas y/o elementos críticos, su fuerza está adosada a las mismas interpretaciones del profesor acerca del contexto en el que se toman las decisiones profesionales; siendo así que llegan a constituir un filón valorativo de la auto-conciencia docente respecto de sus niveles de razonamiento profesional.

Fecunda es ya la relación de términos y conceptos vinculados a esta visión de la reflexión. Con un hilo conductor preferente: el énfasis en la validez fundamental de los juicios de los profesores que se extraen o remiten a sus experiencias profesionales. Y abarcaría, sin pretensión de exhaustividad (ver Sparks-Langer y Bernstein, 1991, p. 42) el estudio de casos centrado en el conocimiento tácito que guía la práctica, el llamado «craft knowledge» en educación y en las prácticas de evaluación de los profesores (cfr. Leinhardt, 1990), el enfoque de la educación y la enseñanza bajo perspectiva artística, la definición de la enseñanza como actuación improvisada, la investigación-acción del profesor y, naturalmente, la indagación narrativa (Connelly y Clandinin, 1990; Clandinin Connelly, 1988), cuya base la forman los relatos escritos, bien por los profesores, o bien sobre los profesores. Huelga remarcar, pues, que en la perspectiva que nos ocupa, el pensamiento reflexivo es sinónimo de narración o relato, con personajes, argumentos y contextos incluidos. Es interesante señalar, de la mano de los mismos Connelly y Clandinin 1990, p. 4), que lo verdaderamente importante cuando empieza la indagación narrativa es que todos los participantes tengan voz. Y citando el trabajo de Elbow (1986) anotan que esto implica jugar al «juego de la creencia», un modo de trabajar dentro de una relación que conecta al conocedor con lo conocido. La distancia o la separación no caracterizan precisamente el saber conectado. El juego de la creencia sería una forma de saber lo que implica un proceso de auto-inserción en el relato del otro como vía para llegar a conocer su relato. Además, el autor enfatiza la naturaleza colaborativa del juego 
escribiendo «el believing game ...es esencialmente cooperativo o colaborativo. El evento central es el acto de afirmar o afectar/entrar en la percepción o el pensamiento de alguien» (p. 289).

Siguiendo el mismo esquema de apartados anteriores, diremos que un tema de unión sobre los elementos narrativos de la reflexión es el circunscrito a los estudios naturalistas. No es ningún secreto que, contrariamente a los trabajos de índole experimental y cuantitativa, estos exploran los significados y las interpretaciones que los profesionales dan a sus vidas normales. De hecho, en los últimos años se ha defendido la oportunidad de la investigación-acción (cfr. Elliot, 1991) como vehículos de buenos relatos en el campo educativo. Tratando de ejemplificar el enfoque, Sparks-Langer y Bernstein (1991) seleccionan algunas aportaciones-tipo. Una es la de Lampert (1990) en torno a la enseñanza de matemáticas en quinto curso, siendo el mismo protagonista estelar. Su meta consistía en procurar un conocimiento de las matemáticas en el aula del modo más parecido a como se llegan a conocer las matemáticas en su ámbito disciplinar. La investigación fue presentada a modo de relato sobre el aprendizaje y el conocimiento de las matemáticas en el «contexto social» del aula, con la conclusión de que los alumnos satisfacían moderadamente sus metas.

Acto seguido mencionan el estudio de Colton et. al. (1989), que formó parte del antes citado Proyecto C.I.T.E.. En este caso, un pequeño grupo de profesores se reunieron durante una semana con el fin de analizar la noción de reflexión docente e intentar llevar a cabo una indagación centrada en su propia práctica. El resultado tuvo que ver con la adquisición de mayores cotas de equilibrio y mayor nivel de significatividad en relación con las posibilidades de cambio y la obsesiva necesidad de dar solución a los problemas de forma inmediata. Finalmente, usando métodos de investigación-acción colaborativa, Canning (1990) daba cuenta del camino seguido en la implicación de los estudiantes de educación en un proceso de descripción y análisis de sus propios intentos en pos de mayor reflexión. No solo escribían sobre experiencias que habían tenido lugar a su alrededor, sino que ellos mismos eran objeto de reflexión. Al parecer, el empleo de preguntas y de 'feedback' de apoyo ayudó a que los sujetos encontrasen gradualmente sus propias 'voces' a medida que entraban abiertamente en la dinámica (auto)reflexiva.

Con todo, por tanto, no es temerario perfilar beneficios potenciales ligados a la narrativa vital y profesional de los docentes; por ejemplo, gracias a su uso y fomento está siendo más fácil acceder a un mejor conocimiento de la motivación general y de los motivos concretos que animan las acciones que emprenden, de cómo las desarrollan, a qué atribuyen sus resultados y qué consecuencias tienen estos en sus iniciativas futuras, etc.; al igual que el acopio de casos y situaciones que pueden proporcionar para ser aprovechadas en la formación pre-servicio y en-servicio; y, por supuesto, el sentido de mejora que casi siempre se deriva en los profesionales como resultado de su auto-indagación. Tal vez, con el aliciente genérico para todo el discurso educativo de estar contribuyendo a generar una agenda, hasta cierto punto nueva, donde 'ubicar' las relaciones teoría-práctica. Prueba de que este espíritu de indagación está echando algunas raices es que nunca tanto se han pedido y buscado (por más que no en todos los casos se hayan encontrado las más felices y operativas) fórmulas de análisis, investigación e interacción colaborativa universidad-escuela (cfr. Eisner, 1988) sobre cuestiones que afectan directa e inequívocamente a la mejora cualitativa del proceso de enseñanza y aprendizaje. Escuchar y examinar atentamente relatos experienciales referidos por los profesionales de la educa- 
ción puede ser una condición para quienes deseen seguir estudiando, y narrando, con rigor y elegancia lo que significa educar y ser educado. Sin perder de vista -permítasenos la licencia- que todo esto será verdaderamente creible si nos disponemos a la creación y re-creación de relatos colaborativos investigadores-profesores, en una dinámica de indagación mutuamente construida y compartida.

\section{REFERENCIAS BIBLIOGRÁFICAS}

ARMALIN, W. D. y Hoove, R. L. (1989). «Field experience as a vehicle for transformation: ideology, education, and reflective practice», Journal of Teacher Education, vol. 40, 2, 42-48.

BERLINER, D.C. (1986). «In pursuit of the expert pedagogue», Educational Researcher, vol. $15,7,5-13$.

BREZINKA, W. (1990). La educación en una sociedad en crisis, Narcea, Madrid.

BROPHY, J. J. y GOOD, T. (1986). «Teacher behavior and student achievement», en Wittrock, M. C. (ed.). Handbook of research on teaching, Macmillan, N.Y., 328-375.

BRown, J. S.; Collins, A. y DuguID, P. (1989). «Situated Cognition and the culture of learning», Educational Researcher, vol. 18,1, January-february, 32-42.

BULLOUGH, R. V. (1989). «Teacher education and teacher reflectivity», Journal of Teacher Education, vol. 40,2, march-april, 15-21.

CALDERHEAD, J. (1983). «Research into teachers' and student teachers' cognitions: exploring the nature of classroom practice», paper presentado a la Reunión Anual de la A.E.R.A., Montreal, Canadá.

CAldERHEAD, J. (ed.) (1987). Exploring teachers' thinking, Cassell, London.

CALDERHEAD, J. (1989). «Reflectivity teaching and teacher education», Teaching and Teacher Education, vol. 5,1, 43-51.

CANNING, C. (1990). «Reflection: out on a limb. An intrapersonal process and the development of voice», Paper presentado a la Reunión Anual de A.E.R.A., Boston.

CARR, W. y KeMmis, S. (1988). Teoría crítica de la enseñanza, Martínez Roca, Barcelona.

Clandinin, D. J. y ConNELly, F. M. (1988). «Studying teachers' knowledge of classrooms: collaborative research, ethics and the negotiation of narrative», The Journal of Educational Thought, vol. 22,2, 269-282.

ClARK, C. M. (1988). «Asking the right questions about teacher preparation: contributions of research on teacher thinking», Educational Researcher, vol. 17,2, 5-11.

Clark, C. M. y LAMPERT, M. (1986). «The study of teacher thinking: implications for teacher education, Journal of Teacher Education, vol. 37,5, 27-31.

Clark, C. M. y Peterson, P. L. (1986). «Teachers' thought processes», en Wittrock, M. (ed.), Handbook of research on teaching, A.E.R.A. and Macmillan, N.Y., 255-296.

Clark, C. M. y YINGER, R. J. (1987). «Teacher planning», en Calderhead, J. (ed.), Exploring teacher' thinking, Cassell Education, London, 84-103. 
ClifT, R.; Houston, W. R.; y PUGACH, M. (eds.) (1990). Encouraging reflective practice: an examination of issues and exemplars. Teachers College Press, New York.

COCHRAN-SMITH, M. y LYTLE, S. L. (1990). «Research on teaching and teacher research: the issues that divide», Educational Researcher, vol. 19,2, 2-11.

Colton, A. B. et. al. (1989). «Collaborative inquiry into developing reflective pedagogical thinking», Action in Teacher Education, vol, 11,3, 44-52.

CONNELly, F. M. y CLANDININ, D. J. (1990). «Stories of experience and narrative inquiry», Educational Researcher, vol. 19,5, june-july, 2-14.

EGAN, K. (1986). Teaching as storytelling, The University of Chicago Press, Chicago.

EISNER, E. W. (1988). «The primacy of experience and the politics of method», Educational Researcher, vol. 17,5, 15-20.

ElBow, P. (1986). Embracing contraries: explorations in teaching and learning, Oxford University Press, Oxford.

ELLIOTT, J. (1990). La investigación-acción en educación, Morata, Madrid.

FENSTERMACHER, G. (1986). «Philosophy of research on teaching: three aspects», en Wittrock, M. C. (ed.). Handbook of research on teaching, Macmillan, N. York, 37-49.

FLODEN, R. E. y KLINZING, H. G. (1990). «What can research on teacher thinking contribute to teacher preparation?. A second opinion», Educational Researcher, vol. 19,5, 15-20.

GAGE, N. L. (1986). Hard gains in the soft sciences: the case of pedagogy, Phi Delta Kappa, Bloomington, Indiana.

GoODMAN, J. (1984). «Reflection and teacher education: a case study and theoretical analysis», Interchange, vol. 15,3, 9-26.

GRINBERG, J. C. (1989). Reflective pedagogical thinking in teachers education, Eastern Michigan University, USA.

HABermas, J. (1982). Conocimiento e interés, Taurus, Madrid.

HABERMAS, J. (1984). Ciencia y tecnología como ideología, Tecnos, Madrid.

HoPKINS, D. (1989). Investigación en el aula, P.P.U., Barcelona.

JACKSON, P. W. (1987). «On the place of narration in teaching», en Berliner, D. y Rosenshine, B. (eds.). Talks to teachers, Random House, N. Y., 307-328.

KILlion, J. P. y Todnem, G. R. (1991). «A process for personal theory building», Educational Leadership, vol. 48, 6, 14-16.

LAMPERT, M. (1990). «When the problem is not the question and the solution is not the answer: mathematical knowing and teaching», American Educational Research Journal, vol. $27,1,29-64$.

LAMPERT, M. y ClARK, C. M. (1990). «Expert knowledge and expert thinking in teaching: a response to Floden and Klinzing», Educational Researcher, vol. 19, 5, 21-24.

Lave, J. (1988). Cognition in practice, Cambridge University Press, Cambridge, Massachussetts.

LEINHARDT, G. (1990). «Capturing craft knowledge in teaching», Educational Researcher, vol. $19,2,18-25$. 
LEINHARDT, G. y GREENo, J. G. (1986). «The cognitive skill of teaching», Journal of Educational Psychology, vol. 78,2, 75-95.

LISTON, D. y ZEICHNER, K. (1987). «Reflective teacher education and moral deliberation», Journal of Teacher Education, vol. 38, 6, 2-8.

MARSICK, V. J. (1991). «Aprendizaje en el centro de trabajo. El caso de la reflexividad y de la reflexividad crítica», Revista de Educación, 294, 141-154.

MCLAREN, P. (1987). «Critical pedagogy and the dream of emancipation», Social Education, vol. 51, 2, 146-150.

MCLAUGHLIN, M. W. (1990). The contexts of teaching in secondary schools. Teachers' Realities, Teachers College Press, N. Y.

MCNAMARA, D. (1991). «Vernacular Pedagogy», British Journal of Educational Studies, vol. XXXIX, 3, august, 297-310.

NisBeTT, R. E. y Ross, L. (1980). Human inference: stretegies and shortcomings of social judgement, Prentice Hall, Englewood Cliffs, N. J.

PASCH, M. et. al. (1990). Teaching as decision making, Longman, New York.

RADL, R. (1991). «La educación como interacción simbólica», Revista de Ciencias de la Educación, 145, 7-14.

Rogoff, B. (1990). Apprenticeship in thinking, Oxford University Press, N. York.

Rogoff, B. y LAVE, J. (ed.) (1984). Everyday cognition: its development in social context, Harvard University Press, Cambridge, Massachussetts, USA.

ROSENSHINE, B. (1986). «Unsolved issues in teaching content: a critique of a lesson on Federalist paper No. 10», trabajo presentado a la Reunión Anual de A.E.R.A., San Francisco.

Rosenshine, B. y STEvens, R. (1986). «Teaching functions», en Wittrock, M. (ed.). Handbook of research on teaching, Macmillan, N. Y., 376-391.

Ross, D. D. (1989). «First steps in developing a reflective approach», Journal of Teacher Education, vol. 40, 2, 22-30.

Ross, D. D. (1990). «Programmatic structures for the preparation of reflective teachers», en Clift, R.; Houston, W. R.; y Pugach, M. D. (eds.). Encouraging reflective practice in education, Teachers College Press, N. Y., 97-118.

Ross, D. D. y KyLE, D. W. (1987). «Helping preservice teachers learn to use teacher effectiveness research appropriately», Journal of Teacher Education, vol. 38, 2, 40-45.

SANTOS REGO, M. A. (1989). «Formación y aprendizaje cooperativo de los profesores: un enfoque experiencial», Revista Galega de Psicopedagoxía, 2, 3, 83-94.

Santos Rego, M. A. (1990). «Estructuras de aprendizaje y métodos cooperativos en educación», Revista Española de Pedagogía, 185, enero-abril, 53-78.

SANTos Rego, M. A. (1990a). «El relato como integrador de procesos educativos», Dársena, 2, enero, 77-83.

SHULMAN, L. S. (1987). «Knowledge and teaching: foundations of the new reform», Harvard Educational Review, vol. 57,1, february, 1-22.

SIMON, R. (1987). «Empowerment as a pedagogy of possibility», Language Arts, vol. 64, 4, 370-382. 
SMYTH, J. (1986). Reflection-in-action, Deakin University Press, Geelong, Australia.

SMYTH, J. (1987). Educating teachers: changing the nature of pedagogical knowledge, Falmer Press, London.

SMYTH, J. (1989). «Developing and sustaining critical reflection in teacher education», Journal of Teacher Education, vol. XXXX, 2, 2-9.

SPARKS-LANGER, G. M. et. al. (1990). «Reflective pedagogical thinking: how can we promote it and measure it», J. of T. Education, vol. 41, 5, 23-32.

Sparks-Langer, G. M. y Bernstein Colton, A. (1991). «Synthesis of research on teachers' reflective thinking», Educational Leadership, vol. 48, 6, 37-44.

SuCHMAN, L. (1987). Plans and situated actions, Cambridge University Press, New York.

SUTTON-SMYTH, B. (1986). «Children's fiction making, en Sarbin, T. R. (ed.). Narrative psychology: the storied nature of human conduct, Praeger, New York, 67-90.

Tom, A. (1985). «Inquiring into inquiry teacher education», trabajo presentado a la Reunión Anual de A.E.R.A., Chicago, April.

USHER, R. S. (1991). «Situación de la educación de adultos en la práctica», Revista de Educación, 294, 155-178.

VAN MANEN, M. (1977). «Linking ways of knowing with ways of being practical», Curriculum Inquiry, 6, 3, 205-228.

WeLlington, B. (1991). «The promise of reflective practice», Educational Leadership, vol. $48,6,4-5$.

WILDMAN, T. et. al. (1987). Teachers learning from teachers: a mentor's guide for supporting beginning teachers, College of Education, Virginia Technology University, Blacksburg.

Wilson, S. M.; SHULMAN, L. S.; y RICHERT, A. (1987). «150 different ways' of knowing: representations of knowledge in teaching», en Calderhead, J. (ed.). Exploring teachers' thinking, Cassell, London, 104-124.

ZeICHNER, K. M. y Liston, D. P. (1987). «Teaching student teachers to reflect», Harvard Educational Review, vol. 57, 1, 23-48.

ZIMMERMAN, B. J. (1989). «A social cognitive view of self-regulated academic learning», Journal of Educational Psychology, vol. 81, 3, 329-339. 